\title{
Eosinophilic meningitis and radiculomyelitis in Thailand, caused by CNS invasion of Gnathostoma spinigerum and Angiostrongylus cantonensis
}

\author{
ERICH SCHMUTZHARD, PRASERT BOONGIRD, ATHASIT VEJJAJIVA
}

From the Department of Neurology, University Hospital Innsbruck, Austria, and the Division of Neurology, Department of Medicine, Faculty of Medicine, Ramathibodi Hospital, Mahidol University, Bangkok, Thailand

SUMMARY During the 6 year period from January 1980 to December 198544 patients with infection of the central nervous system by Gnathostoma spinigerum or Angiostrongylus cantonensis were admitted to the Division of Neurology, Ramathibodi Hospital, Bangkok, Thailand. In 16 patients the diagnosis could be confirmed serologically by means of ELISA techniques. In gnathostomiasis encephalitis, myelitis, radiculitis and subarachnoid haemorrhage formed the majority of clinical syndromes. Intracerebral haematoma and transitory obstructive hydrocephalus are described in this report as being caused by Gnathostoma spinigerum infection for the first time. In angiostronglyus infections the clinical syndrome of meningitis was predominant, but one patient, whose angiostrongyliasis was proved serologically, also showed bilateral paresis of abducens nerve. The main laboratory finding was eosinophilic pleocytosis in the CSF $(>10 \%)$ which in patients originating or returning from South-East-Asia, particularly Thailand, is highly suggestive of these parasitic infections. Increasing transcontinental travel, influx of refugees and those seeking asylum as well as importation of food from South East Asian countries demand greater awareness of these parasitic infections even in Central Europe.

Eosinophilia of the cerebrospinal fluid (CSF) can be found in various parasitic infestations of the central nervous system (CNS), in inflammatory diseases such as tuberculous meningitis, cerebrospinal syphilis, viral and fungal meningitis, in drug allergies, in multiple sclerosis and in neoplasms, like malignant lymphoma, Hodgkin's disease and leukaemia. ${ }^{1}$

In South East Asia parasites are, by far, the commonest cause of eosinophilic pleocytosis in the CSF; in the majority of cases Cysticercus cellulosae, Angiostrongylus cantonensis or Gnathostoma spinigerum represent the responsible agents. Whereas the tapeworm Cysticercus cellulosae has a worldwide distribution, the two nematodes are mainly or almost exclusively found in the region of South East Asia. ${ }^{2} 3$

Address for reprint requests: Erich Schmutzhard, M.D., DTM\&H, Univ.-Klinik für Neurologie, Anichstr. 35, 6020 Innsbruck, Austria.

Received 24 March 1987 and in revised form 27 May 1987. Accepted 1 July 1987
Thailand is one of the most favoured destinations of European travellers; in 1985 more than 100,000 German tourists visited this country. ${ }^{4}$ In recent years more and more (particularly young) travellers spend longer periods in rural areas of this region, living together with local people, trying to imitate local customs and following local eating habits. Thus, European travellers returning from this region might harbour parasites causing little or not understood clinical signs and symptoms. Therefore it seems to be reasonable and justified to delineate the clinical spectrum, the epidemiological features and the diagnostic and therapeutic implications of these two infections.

\section{Gnathostomiasis}

Gnathostoma spinigerum, a tissue nematode of cats and dogs, ${ }^{5}$ was first discovered and described by $\mathrm{Sir}$ Richard Owen in 1836. This parasite was isolated from a tumour of the stomach wall of a tiger at the London Zoo. ${ }^{6}$ Prommas and Daengsvang discovered its life cycle, ${ }^{7}$ which was further elucidated in the late 
'60s. ${ }^{8}$ In 1889 the first human infection with this parasite, then known as Gnathostoma siamense, was diagnosed in a Bangkokian girl. ${ }^{9}$ Nervous system gnathostomiasis, however, has only been recognised for the past 20 years. ${ }^{10}$ Its neuropathological findings and clinical patterns have been presented by Bunnag et al,,$^{11}$ Boongird et al ${ }^{12}$ and Vejjaviva. ${ }^{3}$

Humans may become facultative hosts by eating raw, infected intermediate hosts such as freshwater fish, frogs and snakes. In Thailand at least 36 species of freshwater fish have been found to be infected with the third stage infective larvae. ${ }^{513}$ If intermediate hosts are eaten by paratenic hosts, such as chicken and ducks, the third stage larvae cannot develop further, but they invade the tissues of these hosts. Humans can also be infected when the paratenic hosts are eaten raw or undercooked. In fact, domestic ducks and chickens probably constitute the most important source of human infection. ${ }^{14}$

The worm's head is armed by rows of hooklets and the body contains numerous spines. It's length measures $4-5 \mathrm{~mm}$, its width around $0.5 \mathrm{~mm} .{ }^{5}$ The $3 \mathrm{rd}$ stage larvae cannot complete their cycle in humans, and migrate for a variable time causing irritation and inflammation in various organs. Clinically gnathostomiasis causes migratory swellings, creeping eruption in any organ, the most serious affection being the invasion of the central nervous system. ${ }^{5}$

\section{Angiostrongyliasis}

Nomura and Lin were the first to report the recovery of the third stage larvae of Angiostrongylus cantonenis, the lungworm of rats, from the spinal fluid of a Chinese patient. ${ }^{15}$ The majority of cases have been found in South East Asia and the Pacific islands. ${ }^{2}$ The most comprehensive clinical and epidemiological studies were carried out by Punyagupta et al, ${ }^{1617}$ who presented the symptoms, physical signs and laboratory results of 484 probable angiostrongyliasis patients. The adults, measuring on average $20 \mathrm{~mm}$ in length and $0.3 \mathrm{~mm}$ in width, live in the pulmonary arterioles of rodents, in particular, domestic rats, the definite hosts. ${ }^{17}$ The overall rate of infection of rats by $A$. cantonensis in endemic areas is $1 \cdot 2,{ }^{17}$ most recent data on rat-infection rate in urban Bangkok reach $40.5 \% .^{18}$ Various snails, slugs, prawns and crabs are intermediate hosts. Humans are usually infected by eating infected intermediate hosts, that have not been properly cooked. In Thailand Pila snails are the main source of infections. Punyagupta found this nematode in up to $20 \%$ of such collected snails, which are often served in Thailand as a delicacy together with alcohol. ${ }^{17}$

In humans the parasite does not complete its life cycle and dies, for example, within the brain. Here it provokes a marked inflammatory response, the main clinical manifestation being eosinophilic meningitis. ${ }^{16}$

\section{Patients and methods}

During the 6 year period Janury 1980 to December 1985618 patients suffering from infectious diseases of the CNS were admitted to the Division of Neurology, Ramathibodi Hospital, Bangkok, Thailand. In $44(7 \cdot 1 \%)$ the diagnosis of eosinophilic meningitis, radiculomyelitis or encephalitis had to be established. The categorisation into angiostrongyliasis or gnathostomiasis was done according to the criteria given by Boongird et al ${ }^{12}$ and Punyagupta et al. ${ }^{16}$ Additionally, since 1984 enzyme linked immunosorbent assays (ELISA) for both parasitic diseases were carried out. ${ }^{1920}$ The records of these 44 patients were studied retrospectively with particular regard to epidemiological features, the spectrum of neurological signs and symptoms and diagnostic as well as prognostic aspects. In all patients lumbar puncture was performed; in those presenting with focal cerebral lesions CT of the brain was done. All sediments of CSFs containing more than 20 cells $/ \mathrm{mm}^{3}$ were routinely stained by Giemsa method in order to ensure the detection of eosinophilia in the CSF.

\section{Results}

In 39 patients gnathostomiasis and in five angiostrongyliasis was diagnosed. Beside the above mentioned epidemiological and clinical diagnostic aspects, in 16 cases the diagnosis could be confirmed serologically by means of ELISA techniques.

The age distribution is shown in fig 1 . The youngest patient was 15 years of age and the oldest 74 years, median age being 33 years. The disease was more commonly diagnosed in males than in females, the male/female ratio being $1 \cdot 8 / 1$. The mean duration of hospital admission was 9.5 days (range 2-44 days).

The illness occurred throughout the year, and no seasonal peak could be observed. The patients came from almost the entire country (fig 2), to the northeast from Khon Kaen, to the north from as far as Phitsanulok, from Ubon Ratchathani to the east and as far as Nakkon Si Thammarat in the far south. People of any occupation were among the patients although the farmers constituted the biggest single group with 23 out of $44(52 \%)$.

\section{Gnathostomiasis}

In 39 patients the diagnosis of CNS gnathostomiasis was established. In 14 cases the suspected diagnosis could be confirmed serologically. In two cases the third stage larva could be removed eventually during the course of illness from skin swellings. The remaining 23 were diagnosed according to the criteria given by Boongird et al. ${ }^{12}$

The conditions with which the patients presented are listed in table 1 . Meningitis was the prominent feature in $41 \%$ of our patients, but only in two cases 


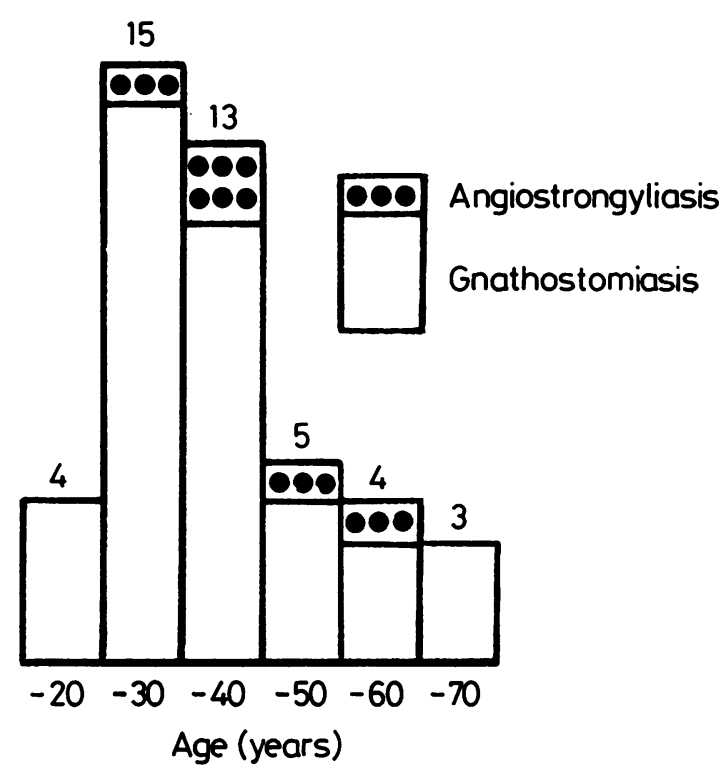

Fig 1 Age distribution $(N=44)$.

was it the only clinical syndrome. In 12 patients it was associated with cranial nerve palsy and/or encephalitis. Encephalitis presented in its usual way with headache, signs and symptoms of raised intracranial pressure and, in several cases, with localising signs such as hemiparesis, hemihypaesthesia and aphasia, the latter creating initial problems in differentiating the disease from herpes simplex encephalitis. Lumbar puncture showing an eosinophilia of $56 \%$ and serological testing (ELISA) clarified this diagnostic uncertainty in this particular patient. Subarachnoid haemorrhage (SAH) is a common syndrome seen in CNS gnathostomiasis, a fact with which western neurologists are completely unfamiliar. In $6 \%$ of all SAH admitted to Ramathibodi Hospital during the period 1980-1985 Gnathostoma spinigerum was incriminated as the causative agent (unpublished data). The onset is typically sudden, resembling in most cases the course of SAH due to ruptured aneurysm. In two cases SAH was accompanied and in one case preceded by radicular and/or myelitic signs and symptoms, thus giving a hint to the possible aetiology of the SAH. Radiculitis, radiculomyelitis and myelitis represent a rather well known clinical course of CNS gnathostomiasis, although it was observed in only less than half of all cases in our group of patients. The onset was usually dramatic and often associated with excruciating radicular pains, closely followed by myotoma paresis and dermatomal sensory disturbance. In several instances, as described in case 1 , radiculitis and myelitis did not occur at the same anatomical level and not at the same time, reflecting the $\frac{0}{Z}$ migration of gnathostoma third stage larva. Optic $\underset{\mathbb{D}}{Z}$ nerve lesions were attributed to raised intracranial $\stackrel{c}{3}$ pressure; in one patient direct involvement of the optic nerve was suspected. Intracranial haemorrhage $\overrightarrow{0}$ and transient obstructive hydrocephalus (both 7 proved by cranial computed tomography) have never been described so far in CNS gnathostomiasis. Cases $\stackrel{5}{=}$ 2 and 4 are examples of these syndromes, which presented in a characteristic way. Thus, in Gnathos.toma spinigerum-endemic areas Giemsa staining of a $\vec{F}$ pathological CSF is advisable, even in seemingly straightforward clinical entities like intracranial hae- $\frac{\bar{\sigma}}{\sigma}$ morrhage and hydrocephalus, to ensure the right $\frac{\bar{\omega}}{\bar{*}}$ diagnosis.

The CSF findings are summarised in table 2. The

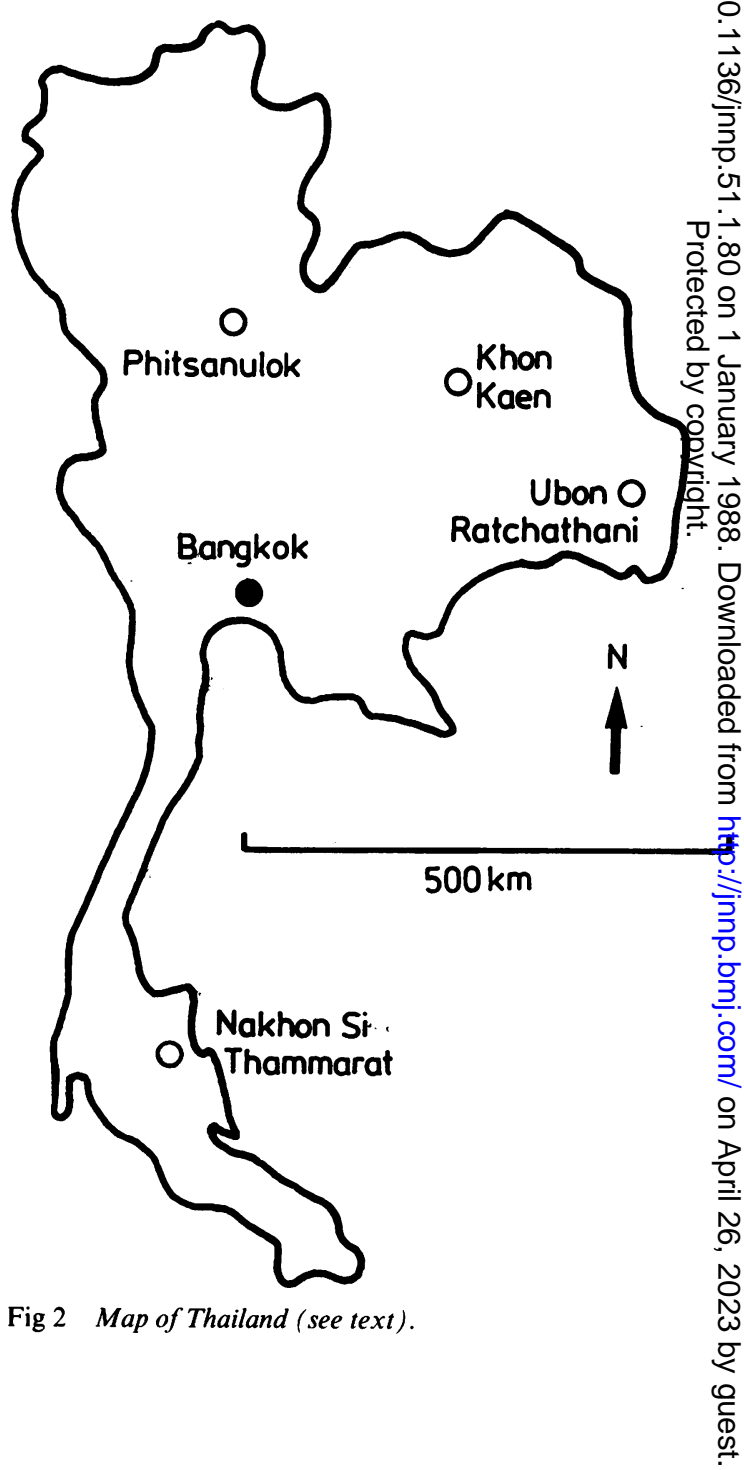


Table 1 Clinical syndromes in CNS gnathostomiasis $(n=39)$

\begin{tabular}{lll}
\hline Syndromes & No of patients & $\%$ \\
\hline Meningitis & 16 & 41 \\
Cranial nerve palsy & 16 & 41 \\
Encephalitis & 12 & $30 \cdot 8$ \\
Subarachnoid haemorrhage & 10 & $25 \cdot 6$ \\
Transverse myelitis & 8 & $20 \cdot 5$ \\
Radiculitis & 6 & $15 \cdot 4$ \\
Optic nerve lesion & 6 & $15 \cdot 4$ \\
Radiculomyelitis & 4 & $10 \cdot 3$ \\
Intracranial haemorrhage & 3 & $7 \cdot 7$ \\
Transient obstructive hydrocephalus & 1 & $2 \cdot 6$ \\
\hline
\end{tabular}

most important single CSF finding was eosinophilia with a median percentage of $54 \%$. No difference in the eosinophilia could be observed if differentiation in non-bloody and bloody CSF was done. Protein content, however, was markedly higher in bloody or xanthochromic than in non-bloody CSF. Although CSF glucose was very low in few cases, this was not a consistent finding in CNS gnathostomiasis. Determination of CSF immunoglobulins and CSF electrophoresis were not possible at Ramathibodi Hospital. Opening pressure was related to the clinical course reflecting raised intracranial pressure.

There was variable blood leucocytosis, median WBC $11750 / \mathrm{mm}^{3}$ (range $\left.5300-28000 / \mathrm{mm}^{3}\right)$ with an eosinophilia of $16 \%(0-47 \%)$, in peripheral blood. Additional laboratory parameters were in most instances normal; only two had slightly pathological liver function tests, five showed moderate anaemia and 14 had raised ESR (more than $20 \mathrm{~mm} /$ first hour). In eight patients CT scan of the skull was performed; three patients showed intracranial haemorrhage, in one obstructive hydrocephalus was observed and four scans did not show any abnormalities. Table 3 shows the outcome. Fifty-four percent $(21 / 39)$ recovered fully, $20.5 \%$ did show some neurological sequelae, whereas $17.8 \%$ did not recover at all from their neurological deficit. The case fatality rate was $7 \cdot 7 \%$ $(3 / 39)$.

\section{Case reports}

Case 1 A 34 year old Thai female farmer from Chayapoon was admitted because of a suddenly developing paraplegia. A few hours before that she had experienced severe burning radicular pains just above the umbilical area. On admission the patient was conscious, she had flaccid paraplegia with a sensory level on Th $8 / 9$ on the right side, to a lesser extent also on the left side at the same level. Vibration sense was impaired. There was incontinence of urine. CSF was slightly turbid, with a pleocytosis of 1200 cells $/ \mathrm{mm}^{3}, 83 \%$ were eosinophils. CSF protein was $0.94 \mathrm{~g} / \mathrm{l}$. CSF/blood sugar ratio was $38 \mathrm{mg} \% / 88 \mathrm{mg} \%$. Because of the high CSF eosinophilia myelogram was judged not necessary and eosinophilic radiculomyelitis was diagnosed. In serum a
Table 2 CSF findings in CNS gnathostomiasis $(\boldsymbol{n}=39)$

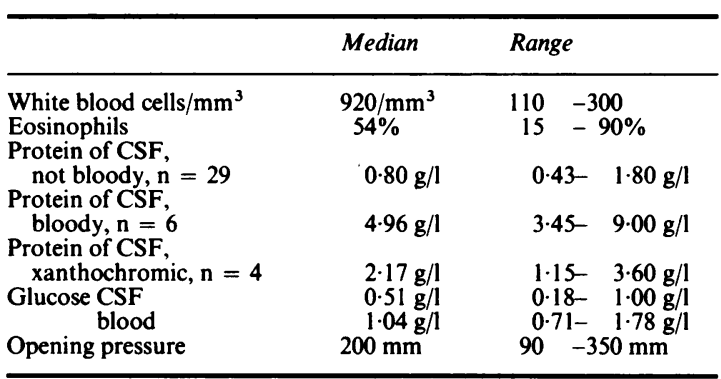

moderate pleocytosis of 13,800 cells $/ \mathrm{mm}^{3}$ was noted, differential count showed $49 \%$ neutrophils, $29 \%$ eosinophils, $20 \%$ lymphocytes and $2 \%$ monocytes. The paraparesis improved partially within the next 4 days, when the patient again developed burning radicular pains involving the cervical roots $\mathrm{C} 5$ and $\mathrm{C} 6$ on the right side. A transient sensory disturbance, and in particular mild paresis of right biceps and deltoid muscles confirmed the radicular lesion. At this time ptosis of the right eyelid and mild miosis was noted, and an incomplete Horner syndrome was diagnosed. All additional laboratory examinations did not reveal any more abnormalities. Because of the progression of disease a therapeutic trial with prednisolone $40 \mathrm{mg}$ daily was started. Slow and incomplete recovery ensued over a period of 3 weeks when she was discharged home.

Case 2 A 39 year old farmer from Pichitr was admitted because of severe throbbing headache, vomiting and weakness of the right extremities. The onset of his symptoms was sudden. On examination the patient was drowsy, showing moderate flaccid right sided hemiparesis. Plantar response was extensor on the right side. In addition, bilateral abducens nerve palsy and right central facial nerve palsy were noted. There was mild neck stiffness. The CSF was slightly turbid, opening pressure $360 \mathrm{~mm}$ and there was pleocytosis with 320 cells $/ \mathrm{mm}^{3}$, of which $80 \%$ were eosinophils, protein was almost normal with $0.55 \mathrm{~g} / \mathrm{l}$ as well as $\mathrm{CSF} /$ blood sugar ratio $(0 \cdot 8 \mathrm{~g} / \mathrm{l} / 1 \cdot 14 \mathrm{~g} / \mathrm{l})$. In blood a mild pleocytosis of 11.700 cells $/ \mathrm{mm}^{3}$ was seen, the differential count being $59 \%$ neutrophils, $23 \%$ eosinophils, $16 \%$ lymphocytes and $2 \%$ monocytes. CT scan showed an intracerebral haemorrhage within the left parietal lobe and with moderate midline shift. The diagnosis of intracranial haemorrhage due to CNS invasion by Gnathostoma spinigerum

Table 3 Outcome (at discharge) and sequelae (1-5 yr later)

(a) Outcome in CNS gnathostomiasis $(n=39)$

Recovered fully

Recovered partially

Not improved

Dead

(b) Long term sequelae in CNS gnathostomiasis $(n=15)$

Paraplegia

Radicular lesion

Cranial nerve lesion

Organic psychosyndrome

Hemiparesis 
was established; epidemiological aspects (the patient had been exposed several times to raw or undercooked fish within the preceding month) and CSF-eosinophilia being strongly suggestive for it. The patient was put on steroids, and gradual improvement ensued. He could be discharged with nearly normal neurological status.

Case 3 A 20 year old farmer from Suin developed 10 days after ingestion of raw fish severe radicular pains $\mathrm{C5}-\mathrm{C} 7$ on the left side. Accordingly paresis of the deltoid, biceps and triceps muscles set in within 24 hours. The dermatomes C5-C7 became hypaesthetic. One day later this polyradicular syndrome was followed by extremely severe headache, vomiting and drowsiness. On admission he had extreme stiffness of the neck, and he complained of nausea. There was horizontal nystagmus, the cranial nerves were otherwise normal. On the left upper extremity a definite polyradicular syndrome C5-C7 was present. No signs of spinal involvement could be detected. Lumbar puncture showed a bloody CSF with an opening pressure of $280 \mathrm{~mm}$ of water, $740 \mathrm{WBCs} / \mathrm{mm}^{3}$ (35\% eosinophils). CSF sugar was $0.36 \mathrm{~g} / \mathrm{l}$, blood sugar $0.95 \mathrm{~g} / \mathrm{l}$. There was mild blood leucocytosis with $13 \%$ eosinophils. CNS gnathostomiasis with radiculitis and subarachnoid haemorrhage was diagnosed. Only slow but steady recovery could be observed. Finally the patient was discharged with mild paresis in the myotomas C5 and C6 (left deltoid and biceps muscles). Neurological condition 3 months later, however, was already almost normal.

Case 4 A 34 year old farmer from Lopburi was admitted with a 3 week history of headache and intermittent diplopia. For several days prior to admission he had suffered from nausea and he had vomited three times. Slight unsteadiness of gait was noted only a few hours before hospitalisation. On examination he showed partial ptosis of the right eyelid, the pupils were anisocoric (the right pupil was wider than the left), the bulbi were divergent, the patient being unable to produce convergence owing to weakness of the right internal rectus muscle. Upward gaze was completely impaired bilaterally. No other cranial nerves were involved. A mild spastic hemiparesis and hemihypaesthesia was present on the right side, plantar response being extensor. The gait was ataxic. Incontinence of urine was denied. CSF contained 120 $\mathrm{WBC} / \mathrm{mm}^{3}$ (39\% eosinophils), opening pressure was high with $380 \mathrm{~mm}$ water. CSF protein and sugar were normal. Only mild peripheral eosinophilia $(9 \%)$ could be observed. CT scan showed deformation of the left thalamic region with compression of the lateral 3rd ventricle. This lesion was clearly enhancing after intravenous administration of contrast medium. Moreover, a moderate hydrocephalus was diagnosed. Follow-up CT scan, 10 days later, still showed partially the lesion in the right thalamic region; the width of lateral ventricles, however, was normal. Antibodies to gnathostoma spinigerum were detected in serum by means of ELISA technique. A course of corticosteroid was started, and the patient's condition improved markedly within 3 weeks.

These four case reports describe on the one hand the main signs and symptoms, such as radiculitis, transverse myelitis, cranial neuritis, meningitis, encephalitis and subarachnoid hemorrhage. Intracerebral haematoma (case 2) and transitory obstruc- tive hydrocephalus (case 4 ), however, have not yet been reported so far as a symptom or complication of CNS gnathostomiasis.

\section{Angiostrongyliasis}

Only five patients suffering from angiostrongyliasis were found in this study. In two cases the diagnosis was confirmed by ELISA, the other three patients fulfilled the criteria given by Punyagupta et al. ${ }^{16}$ In particular, the history of eating Pila snails suggested an infection by Angiostrongylus cantonensis. The number of patients is too small to allow detailed statistical analysis. All five presented with the signs and symptoms of meningitis, in addition, one $\mathbf{4 0}$ year old male with positive $A$. cantonensis titre (by ELISA) presented with bilateral abducens nerve palsy and papilloedema of the left eye. No death occurred in this group.

Interestingly, only one of the five patients was a farmer (from Phitsanulok). Three were employees and one worked as a mason. Two of the employees were residents of Bangkok, but both were born in Ubol, where they frequently went for holidays and family affairs. The mason was a resident of this city, too. The fifth patient was a resident from Nakhon $\mathrm{Si}$ Thammarat in the far south of the country.

CSF analysis showed median pleocytosis of $2039 / \mathrm{mm}^{3}$ with a range of $840-3 \cdot 750 / \mathrm{mm}^{3}$. Median CSF eosinophilia was $71 \%$ (range $35-85 \%$ ). Protein was only slightly elevated, $0.68 \mathrm{~g} / \mathrm{l}$ (range $0.45-1.25$ $\mathrm{g} / \mathrm{l}$ ). CSF sugar was low, $0.049 \mathrm{~g} / \mathrm{l}$ (range 0.32-0.62 $\mathrm{g} / \mathrm{l})$ whereas the median blood sugar was mildly raised, $1.26 \mathrm{~g} / \mathrm{l}$ (range $0.98-1.46 \mathrm{~g} / \mathrm{l}$ ). Opening pressure was on average $280 \mathrm{~mm}$ (range $210-360 \mathrm{~mm}$ ). Blood leucocyte count was 13200 (range 9800-18400), peripheral blood eosinophilia was $10 \%$ (range 3-23\%). No other abnormal laboratory findings could be detected.

\section{Case reports}

Case 1 A 23 year old male Thai employee presented with a history of severe headache, mild fever and vomiting for 2 days. The patient had eaten Pila snails on two occasions during the previous week. Neurological examination showed moderate neck stiffness with positive Kernig sign. There were no motor deficits or sensory disturbances. On admission the patient complained of mild photophobia and nausea. Lumbar puncture revealed pleocytosis with 2685 cells $/ \mathrm{mm}^{3}, 85 \%$ being eosinophils, opening pressure was 292 $\mathrm{mm}$, CSF protein $0.49 \mathrm{~g} / \mathrm{l}$. CSF/blood sugar ratio was $0 \cdot 32 / 1 \cdot 19 \mathrm{~g} / \mathrm{l}$. Blood leucocyte count was $15,700 / \mathrm{mm}^{3}$ with an eosinophilia of $23 \%$. No other abnormal laboratory parameters were detected. The patient was confined to bedrest and put on a 4 day course of prednisolone. The course of disease was uneventful and the patient was discharged without sequelae only 5 days after admission.

Case 2 A 40 year old male Bangkok resident was admitted 
to our hospital only 2 days after returning from a brief holiday in his hometown Ubol. For farewell he had been served alcoholic beverages and Pila snails. He complained of sudden onset of severe headache, associated with nausea. There was no fever. A few hours before admission he developed double vision; he was not able to state on which side this double vision had started. On examination the patient was afebrile, conscious and oriented. Mild neck stiffness was present. The most prominent feature was palsy of both abducens nerves. There was slight papilloedema on the left side. No localising signs could be found. CSF showed pleocytosis of $840 / \mathrm{mm}^{3}$ cells with $35 \%$ eosinophils, a protein content of $0.53 \mathrm{~g} / \mathrm{l}$ and an opening pressure of $360 \mathrm{~mm}$. $\mathrm{CSF} /$ blood sugar ratio was $0 \cdot 62 / 1 \cdot 46 \mathrm{~g} / \mathrm{l}$. EEG and CT-scan were without abnormal findings. Peripheral eosinophilia was $16 \%$, blood leucocytosis $13,800 / \mathrm{mm}^{3}$. ELISA for angiostrongylus antibodies was strongly positive, for gnathostoma antibodies it was negative. The patient was put on prednisolone $40 \mathrm{mg} /$ day. Because the abducens palsy resolved only slowly steroid therapy was continued for 11 days before tapering off. The patient was discharged 20 days after admission with mild diplopia. Half a year later he was seen again at the outpatient department, subjectively feeling well; on careful neurological examination mild left abducens palsy could still be detected.

\section{Discussion}

Many helminths have been reported to be able to invade the CNS and cause a wide variety of neurological signs and symptoms. The various genera are listed in table 4. Those occurring in Thailand are marked with an asterisk. Cysticercosis ${ }^{30}$ and paragonimiasis $^{27}$ as well as schistosomiasis ${ }^{28}$ are characterised by distinct clinical features, mainly presenting as subacute or chronically developing space occupying lesions. Disseminated strongyloidiasis is a known complication of immunosuppressive therapy in patients with intestinal infections with the nematode Strongyloides stercoralis. Neurological involvement ranges from mental status changes to coma; frequently, a concomitant gram-negative bacillary meningitis is observed. ${ }^{21}$ CSF abnormalities of these four parasitic infections are uncharacteristic, eosinophilic pleocytosis in CSF is rarely seen, whereas peripheral eosinophilia is common in early stages of these diseases before CNS involvement has set in. ${ }^{32}$ Toxocariasis, trichinosis and meningoencephalitis due to the racoon ascarid $B$. procyonis are frequently associated with high CSF eosinophilia but are not endemic in South East Asia.

Thus, the finding of eosinophilic pleocytosis $(>10 \%)$ in a patient originating or returning from South East Asia, particularly Thailand, is suggestive of $G$. spinigerum or A. cantonensis infection.

In those cases, in which the serological supportive diagnostic tool of ELISA had been applied, the
Table 4 Helminths potentially invading the CNS

\author{
Nematodes \\ Gnathostoma spinigerum* \\ Angiostrongylus cantonensis* \\ Strongyloides stercoralis ${ }^{21 *}$ \\ Trichinella spiralis ${ }^{22}$ \\ Toxocara canis ${ }^{23}$ \\ Lagochilascaris minor ${ }^{24}$ \\ Baylisascaris procyonis 25 \\ Anisakis spp ${ }^{26}$ \\ Trematodes \\ Paragonimus westermanni ${ }^{27 *}$ \\ $P$. mexicanus \\ Schistosoma haematobium \\ S. japonicum \\ S. mansoni ${ }^{28}$ \\ Cestodes \\ Echinococcus granulosus ${ }^{29}$ \\ E. multilocularis \\ Cysticercus cellulosae ${ }^{30 *}$ \\ Spirometra mansonoides ${ }^{31 *}$
}

*Of medical importance in Thailand

differential diagnostic clinical aspects, given by Punyagupta $e t a l^{16}$ and Boongird $e t a l^{12}$ could be fully confirmed. Radiculitis, radiculomyelitis, cranial nerve involvement, encephalitis and subarachnoid haemorrhage were by far the most frequently observed clinical syndromes in gnathostomiasis which is potentially life threatening. Usually, death is precipitated by invasion of the worm into vital areas of the brainstem or medulla oblongata, ${ }^{11}{ }^{12}$ which was supposedly the case in our three patients although necropsy was not allowed. No death in our series could be attributed to subarachnoid haemorrhage. Only $54 \%$ of the gnathostomiasis patients survived without sequelae. Thirty eight per cent did recover with various degrees of neurological long term sequelae. The deficits comprised hemiparesis, cranial nerve palsy, organic psychosyndrome, radicular lesion, and particularly frequently, paraplegia. Thus, initial transverse myelitis and acute radiculitis bear a high risk of permanent damage. Necropsy reports suggest direct mechanical and inflammatory damage by the migrating worm, in rare cases causing thrombosis of blood vessels and, thus, secondary infarction of spinal cord or brain tissue. ${ }^{1112}$

The main laboratory finding was the dramatic eosinophilic pleocytosis in CSF which did not correspond to the peripheral eosinophilia. Thus, in patients presenting with the above listed signs and symptoms, and originating from South East Asia, it is necessary to stain CSF sediment by the Giemsa method in order not to fail to detect the eosinophilia.

Epidemiological factors support the diagnosis of gnathostomiasis and angiostrongyliasis. Most patients were not inhabitants of Bangkok, being farmers. Thus, obviously, consuming undercooked or 
raw fish, which is practised in many rural areas of Thailand, contributes more to the acquisition of the disease, than the occupation or residence per se. Furthermore, some of these uncooked meals are served as delicacies together with alcoholic beverages. This is a possible explanation for the male preponderance and dominance of the age groups of $20-40$ years.

In angiostrongyliasis frank meningitis is the dominant clinical syndrome. Only one patient showed cranial nerve involvement. Neither spinal cord nor intracerebral lesions were seen in patients with this infection. The outcome was favourable in all cases. These results correspond with the extensive study of 484 angiostrongyliasis patients described by Punyagupta et al. ${ }^{16}$ The CSF data showed eosinophilic pleocytosis with mildly increased protein.

The difference in clinical presentation of these two parasitic diseases is obvious. Serologic examination ${ }^{1920}$ enables us to differentiate them, when neurological signs and symptoms are not clear cut.

There is no specific therapy for either parasitic infestation at the present time. 2,6 diiodo-4 nitrophenol (Ancylol) has shown some beneficial effect in cats with feline Gnathostoma spinigerum infection, when given subcutaneously in repeated doses. ${ }^{33}$ No reports deal, so far, with its applications in humans. Thiabendazole has been used in early stages of migration of larvae of $A$. cantonensis, but this drug fails as soon as the worm reaches the central nervous system. $^{32}$

Corticosteroids have been proven in some instances to be effective, particularly in relieving focal neurologic deficits; it is thought that the perifocal oedema might be reduced by this drug and therefore enhance reduction of neurologic deficits. It is obvious that all supportive therapeutic measures should be applied in patients suffering from neurological disease.

The abundance and variety of intermediate hosts are the main cause of difficulty in controlling parasitic infestations, such as these two described species. Prevention depends upon avoidance or adequate cooking of foods such as freshwater fish, snails, chicken and ducks. Theoretically these measures seem to be very easy to introduce into society but it has proved extremely difficult to apply them on a practical basis. The acceptance of health educational proposals imply change of lifestyles and methods of food preparation. Health education is fundamental for success in eliminating parasitic disease.

Diagnoses of gnathostomiasis and angiostrongyliasis are extremely rare events in Western Europe. Increasing transcontinental travel, influx of refugees and those seeking asylum as well as importation of food from South East Asian countries demand greater awareness of these parasitic infections even in Central Europe.

We are very grateful to Miss Sigrid Permoser for her secretarial expertise.

\section{References}

1 Fishman RA. Cerebrospinal Fluid in Diseases of the Nervous System. London: WB Saunders, 1980:183-4.

2 Smit AM. Eosinophilic meningitis. Trop Geogr Med 1963;15:225-32.

3 Vejjajiva A. Parasitic diseases of the nervous system in Thailand. Clin Exp Neurol 1978;15:92-7.

4 The Nation, Thailand's English-language newspaper. 24th Sept. 1986:25.

5 Daengsvang S. A Monograph on the Genus Gnathostoma and Gnathostomiasis in Thailand. Publication Nr. 21. Tokyo: Southeast Asian Medical Information Center, 1980.

6 Owen R. Gnathostoma spinigerum n.sp. Proc zool Soc (Lond.) Part 4: 1836:123-6.

7 Prommas C, Daengsvang S. Preliminary report of a study on the lifecycle of Gnathostoma spinigerum. J Parasitol 1933;19: 287-92.

8 Daengsvang $\mathrm{S}$. Further observations on the experimental transmission of Gnathostoma spinigerum. Ann Trop Med Parasitol 1968;62:88-94.

9 Levinsen CMG. Om en ny Rundom hos mennesket Cheiracanthus siamensis n. sp. Med Dansk, Naturh Foren Kobenhavno 1889:323-6.

10 Chitanondh H, Rosen L. Fatal eosinophilic encephalomyelitis caused by the nematode Gnathostoma spinigerum. Am J Tropo Med Hyg 1967;16:638-45.

11 Bunnag T, Corner DS, Punyagupta S. Eosinophilic myeloencephalitis caused by Gnathostoma spinigerum. Neuropathology of nine cases. J Neurol Sci 1970;10:419-34.

12 Boongird P, Phuapradit $P$, Siridej N, Chirachariyavej $T$ Chuahirun S, Vejjajiva A. Neurological manifestations of gnathostomiasis. J Neurol Sci 1977;31:279-91.

13 Daengsvang S, Papasarathorn T, Chulalerk U, Tongkoom B. Epidemiologic observations on Gnathostoma spinigerum in Thailand. J Trop Med Hyg 1964;67:144-7.

14 Daengsvang S, Thienprasitthi P, Chomcherngpat P. Furthe investigations of natural and experimental hosts of larvae of Gnathostoma spinigerum in Thailand. Am J Trop Med Hyg 1966;15:727-9.

15 Nomura L, Lin PH. First case report of human infection with Haemostrongylus ratti. Yukogawa Taiwan No Ikai 1945;3 589-91.

16 Punyagupta S, Juttijudata P, Bunnag T. Eosinophilic meningitis in Thailand. Clinical studies of 484 typical cases probably caused by Angiostrongylus cantonensis. Am J Trop Med Hyg 1975;24:921-31.

17 Punyagupta S, Bunnag T, Juttijudata P, Rosen L. Eosinophilic meningitis in Thailand. Epidemiologic studies of 484 typical cases and the etiologic role of Angiostrongylus cantonensis. Am J Trop Med Hyg 1970;19:950-8.

18 Hinz E. Angiostrongylus Befall bei Wanderratten in Bangkok, Thailand. Mitt Österr Ges Tropenmed Parasitol 1986;8:83-8.

19 Dharmkrong-At A, Migasena S, Suntharasomai P, Bunnag D Priwan R, Sirisinha S. Enzyme-linked immunosorbent assay for detection of antibody to gnathostoma antigen in patients with intermittent cutaneous migratory swelling. J Clin Microbiol 1986;23:847-51.

20 Ko RC, Chiu MC, Kum W, Chan SH. First report of human angiostrongyliasis in Hongkong diagnosed by computerized axial tomography (CAT) and enzyme linked immunosorbent assay. Trans $R$ Soc Trop Med Hyg 1984;78:354-5. 
21 Scowden EB, Schaffner W, Stone WJ. Overwhelming strongyloidiasis: an unappreciated opportunistic infection. Medicine 1978;57:527-44.

22 Most $\mathrm{H}$, Abeles MM. Trichiniasis involving the nervous system. Arch Neurol Psychiatr 1937;37:589-616.

23 Woodruff AW. Toxocariasis. Br Med J 1970;2:663-9.

24 Rosenberg S, Lopes MBS, Masuda Z, Campos R, Vieria Bressan MCR. Fatal encephalopathy due to Lagochilascaris minor infection. Am J Trop Med Hyg 1986;35:575-8.

25 Fox AS, Boyer KM. Fatal eosinophilic meningoencephalitis and visceral larva migrans caused by the racoon ascarid Baylisascaris procyonis. $N$ Engl J Med 1985;312:1619-23.

26 Khalil LF. Larval nematodes in the herring (Clupea harengus) from British coastal waters and adjacent territories. $J$ Marine Biol Assoc UK 1969;49:641-59.

$27 \mathrm{Oh}$ SJ. Cerebral paragonimiasis. Trans Am Neurol Assoc
1967;92:275-7.

28 Scrimgeour EM, Gajdusek DC. Involvement of the central nervous system in Schistosoma mansoni and S. haematobium infection. Brain 1985;108:1023-38.

29 McCorkell SJ, Lewall DB. Computed tomography of intracerebral echinococcal cysts in children. J Comp Assist Tomogr 1985;9:514-8.

30 McGormic GF, Zee CS, Heiden J. Cysticercosis cerebri, review of 127 cases. Arch Neurol 1982;39:534-9.

31 Fan KJ, Pezeshkpour GH. Cerebral sparganosis. Neurology 1986;36:1249-51.

32 Bia FJ, Barry M. Parasitic infections of the central nervous system. Neurol Clin 1986;4:171-206.

33 Daengsvang S. Chemotherapy of feline Gnathostoma spinigerum migrating stage with multiple subcutaneous doses of ancylol. SE As J Trop Med Publ H 1980;11:359-62. 